\title{
Beyond-Birthday-Bound Security Based on Tweakable Block Cipher
}

\author{
Kazuhiko Minematsu \\ NEC Corporation, 1753 Shimonumabe, Nakahara-Ku, Kawasaki, Japan \\ k-minematsu@ah.jp.nec.com
}

\begin{abstract}
This paper studies how to build a $2 n$-bit block cipher which is hard to distinguish from a truly random permutation against attacks with $q \approx 2^{n / 2}$ queries, i.e., birthday attacks. Unlike previous approaches using pseudorandom functions, we present a simple and efficient proposal using a tweakable block cipher as an internal module. Our proposal is provably secure against birthday attacks, if underlying tweakable block cipher is also secure against birthday attacks. We also study how to build such tweakable block ciphers from ordinary block ciphers, which may be of independent interest.
\end{abstract}

Keywords: Block Cipher Mode, Birthday Bound, Tweakable Block Cipher.

\section{Introduction}

A double-block-length cipher (DBLC), i.e. a $2 n$-bit block cipher made from $n$-bit block components, has been one of the main research topics in the symmetric cryptography. In particular, a seminal work of Luby and Rackoff [17] proved that a 4-round Feistel permutation is computationally hard to distinguish from a truly random permutation if each round function is an $n$-bit pseudorandom function [11. The proof of [17] is valid for chosen-ciphertext attacks (CCAs) using $q \ll 2^{n / 2}$ queries, and is called a proof of $O\left(2^{n / 2}\right)$-security. As $2^{n / 2}$ is related to the birthday paradox for $n$-bit variables, it is also called the security up to the birthday bound (for $n$ ). Then, building a DBLC having beyond-birthday-bound security, i.e., $O\left(2^{\omega+n / 2}\right)$-security for some $\omega>0$, is an interesting research topic from theoretical and practical aspects. In particular, such a DBLC can improve the security of any block cipher mode that has $O\left(2^{n / 2}\right)$-security with an $n$-bit block cipher1 1 . However, achieving $O\left(2^{\omega+n / 2}\right)$-security is generally difficult, even for a small $\omega$. We have very few known DBLC proposals having this property. All of them were based on Feistel permutations using pseudorandom functions 22 18 20. Although these studies indicated the great potential of Feistel permutation, we wondered if using Feistel was the only solution.

In this paper, we demonstrate how this problem can be solved using a tweakable block cipher, defined by Liskov et al.[16. In particular, we present

${ }^{1}$ For some specific applications, such as stateful encryption and stateful authentication, block cipher modes with beyond-birthday-bound security are known 15 . 
how to build a DBLC based on a tweakable block cipher $\widetilde{E}$ with $n$-bit block and $m$-bit tweak for any $1 \leq m \leq n$, and prove $O\left(2^{(n+m) / 2}\right)$-security against CCAs. One significant fact is that it is optimally efficient, as it requires only two $\widehat{E}$ calls (independently of $m$ ) and some universal hash functions. Thus, assuming very fast universal hash functions (e.g., 25]), our DBLC will have almost the same throughput as that of a tweakable block cipher. This means that, the task of building a secure $2 n$-bit block cipher can be efficiently reduced to that of building a secure $n$-bit block tweakable block cipher. We think this is an interesting application of tweakable block cipher, that has not been mentioned before. As a by-product, we provide some variants such as a pseudorandom function with $2 n$-bit input and $n$-bit output. All variants are optimally efficient in the sense defined above.

We have to emphasize that the birthday bound here is with respect to $n$, and not to $n+m$. The security of our scheme is still up to the birthday bound of input length of the cryptographic primitive (as with Yasuda 28]). Although this makes the problem much easier in general, our result is still non-trivial and highly optimized as a solution to beyond-birthday-bound security for $n$.

As our DBLC requires a tweakable block cipher with beyond-birthday-bound security, we also discuss how to realize it. Specifically, we focus on constructions using $n$-bit block ciphers. Although known constructions [16] 24] are only $O\left(2^{n / 2}\right)$-secure, we provide a simple solution using tweak-dependent key changes with a concrete security proof. Unfortunately, this scheme is only the first step: it can be very slow and has some severe theoretical limitations, thus is far from being perfect. Building a better scheme remains an interesting future direction of research.

\section{Preliminaries}

\subsection{Basic Notations}

A random variable will be written in capital letters and its sampled value will be written in the corresponding small letters. Let $\Sigma^{n}$ denote $\{0,1\}^{n}$. The bit length of a binary sequence $x$ is denoted by $|x|$, and $x_{[i, j]}$ denotes a subsequence of $x$ from $i$-th to $j$-th bit, for $1 \leq i<j \leq|x|$. A uniform random function (URF) with $n$-bit input and $\ell$-bit output, denoted by $\mathrm{R}_{n, \ell}$, is a random variable uniformly distributed over $\left\{f: \Sigma^{n} \rightarrow \Sigma^{\ell}\right\}$. Similarly, a random variable uniformly distributed over all $n$-bit permutations is an $n$-bit block uniform random permutation (URP) and is denoted by $\mathrm{P}_{n}$. If $F_{K}: \mathcal{X} \rightarrow \mathcal{Y}$ is a keyed function, then $F_{K}$ is a random variable (not necessarily uniformly) distributed over $\{f: \mathcal{X} \rightarrow \mathcal{Y}\}$. If $F_{K}$ is a keyed permutation, $F_{K}^{-1}$ will denote its inversion. We will omit $K$ and write $F: \mathcal{X} \rightarrow \mathcal{Y}$, when $K$ is clear from the context.

A tweakable block cipher [16] is a keyed permutation with auxiliary input called tweak. Formally, a ciphertext of a tweakable blockcipher, $\widetilde{E}: \mathcal{M} \times \mathcal{T} \rightarrow \mathcal{M}$, is $C=\widetilde{E}(M, T)$, where $M \in \mathcal{M}$ is a plaintext and $T \in \mathcal{T}$ is the tweak. The encryption, $\widetilde{E}$, must be a keyed permutation over $\mathcal{M}$ for every $T \in \mathcal{T}$, and the decryption is defined as $\widetilde{E}^{-1}(C, T)=M$ with $\widetilde{E}^{-1}: \mathcal{M} \times \mathcal{T} \rightarrow \mathcal{M}$. If $\widetilde{E}$ has $n$-bit 
block and $m$-bit tweak, we say it is an $(n, m)$-bit tweakable cipher. An $(n, m)$-bit tweakable URP is the set of $2^{m}$ independent URPs (i.e., an $n$-bit URP is used for each $m$-bit tweak) and is denoted by $\widetilde{\mathrm{P}}_{n, m}$. We write $\widetilde{\mathrm{P}}_{n}$ if $m$ is clear from the context.

\subsection{Security Notion}

Consider the game in which we want to distinguish two keyed functions, $G$ and $G^{\prime}$, using a black-box access to them. We define classes of attacks: chosenplaintext attack (CPA), and chosen-ciphertext attack (CCA), and their tweaked versions, i.e., a tweak and a plaintext (or ciphertext) can be arbitrarily chosen. Here, (tweaked) CCA can be defined when $G$ and $G^{\prime}$ are (tweakable) permutations. Let atk $\in\{\mathrm{cpa}, \mathrm{cca}, \widetilde{\mathrm{cpa}}, \widetilde{\mathrm{cca}}\}$, where $\widetilde{\mathrm{cpa}}(\widetilde{\mathrm{cca}})$ denotes tweaked CPA (CCA). The maximum advantage of adversary using atk in distinguishing $G$ and $G^{\prime}$ is:

$$
\operatorname{Adv}_{G, G^{\prime}}^{\text {atk }}(\theta) \stackrel{\text { def }}{=} \max _{\mathcal{D}: \theta-\text { atk }}\left|\operatorname{Pr}\left[\mathcal{D}^{G}=1\right]-\operatorname{Pr}\left[\mathcal{D}^{G^{\prime}}=1\right]\right|,
$$

where $\mathcal{D}^{G}=1$ denotes that $\mathcal{D}$ 's guess is 1 , which indicates $G$ or $G^{\prime}$. The parameter $\theta$ denotes the attack resource, such as the number of queries, $q$, and time complexity [11, $\tau$. If $\theta$ does not contain $\tau$, the adversary has no computational restriction. The maximum is taken for all atk-adversaries having $\theta$. For $G: \Sigma^{n} \rightarrow \Sigma^{m}$, we have

$$
\operatorname{Adv}_{G}^{\text {prf }}(\theta) \stackrel{\text { def }}{=} \operatorname{Adv}_{G, \mathrm{R}_{n, m}}^{\text {cpa }}(\theta), \operatorname{Adv}_{G}^{\text {sprp }}(\theta) \stackrel{\text { def }}{=} \operatorname{Adv}_{G, \mathrm{P}_{n}}^{\text {cca }}(\theta), \operatorname{Adv}_{G}^{\text {prp }}(\theta) \stackrel{\text { def }}{=} \operatorname{Adv}_{G, \mathrm{P}_{n}}^{\text {cpa }}(\theta),
$$

where the last two equations are defined if $G$ is an $n$-bit permutation, Moreover, if $G$ is an $(n, m)$-bit tweakable cipher, we define

$$
\operatorname{Adv}_{G}^{\widetilde{\text { pprp }}}(\theta) \stackrel{\text { def }}{=} \operatorname{Adv}_{G, \widetilde{\mathrm{P}_{n}}}^{\widetilde{\mathrm{P} a}}(\theta), \text { and } \operatorname{Adv}_{G}^{\widetilde{\mathrm{prp}}}(\theta) \stackrel{\text { def }}{=} \operatorname{Adv}_{G, \widetilde{\mathrm{P}}_{n, m}}^{\widetilde{\mathrm{ppa}}}(\theta)
$$

If $\operatorname{Adv}_{G}^{\mathrm{prf}}(\theta)$ is negligibly small for all practical $\theta$ (the definition of "practical $\theta$ " depends on users.), $G$ is a pseudorandom function (PRF)[11]. If $G$ is invertible, it is also called a pseudorandom permutation (PRP). In addition, if $\operatorname{Adv}_{G}^{\text {sprp }}(\theta)$ is negligibly small, $G$ is a strong pseudorandom permutation (SPRP). If $G$ is a tweakable cipher, tweakable SPRP and PRP are similarly defined using $\operatorname{Adv}_{G}^{\widehat{\text { sprp }}}(\theta)$ and $\operatorname{Adv}_{G}^{\widehat{\text { prp }}}(\theta)$. Generally, we say $G$ is secure if $\operatorname{Adv}_{G}^{\widehat{\text { sprp }}}(\theta)$ is negligibly small.

CCA-CPA conversion. In our security proof, it is convenient to use a conversion from a cca-advantage into a cpa-advantage. For this purpose, we introduce a subclass of cpa called $\mathrm{cpa}^{\prime}$, which is as follows. First, for any keyed permutation $G$ over $\mathcal{M}$, let $\langle G\rangle: \mathcal{M} \times \Sigma \rightarrow \mathcal{M}$ be the equivalent representation of $G$, where $\langle G\rangle(x, 0)=G(x)$ and $\langle G\rangle(x, 1)=G^{-1}(x)$. This expression also holds true for tweakable permutations, i.e., for any tweakable permutation $\widetilde{G}$ with message space $\mathcal{M}$ and tweak space $\mathcal{T},\langle\widetilde{G}\rangle$ is an equivalent keyed function $: \mathcal{M} \times \mathcal{T} \times \Sigma \rightarrow \mathcal{M}$. The LSB of a query to $\langle G\rangle$ is called a operation indicator. 
Consider $F: \mathcal{M} \times \Sigma \rightarrow \mathcal{M}$ and a cpa-adversary $\mathcal{D}$ interacting with $F$. Let $X_{i}=\left(M_{i}, W_{i}\right) \in \mathcal{M} \times \Sigma$ be the $i$-th query of $\mathcal{D}$ and let $Y_{i} \in \mathcal{M}$ be the $i$-th answer. For any $\mathcal{D}$, we assume $M_{i} \neq M_{j}$ always holds when $W_{i}=W_{j}$ with $i<j$. Moreover, if $Y_{i} \neq M_{j}$ holds whenever $W_{i} \neq W_{j}$ holds with $i<j, \mathcal{D}$ is said to follow the invertibility condition. A cpa-adversary following the invertibility condition is called a cpa'-adversary. If $F$ corresponds to $\langle G\rangle$ for a keyed permutation $G$, violating the invertibility condition is clearly pointless, as outputs are predictable. Thus any cca-adversary avoiding useless queries for $G$ can be simulated by a cpa'-adversary interacting with $\langle G\rangle$. In other words, for any keyed permutations, $E$ and $G$, we have

$$
\operatorname{Adv}_{E, G}^{\mathrm{cca}}(q, \tau)=\operatorname{Adv}_{\langle E\rangle,\langle G\rangle}^{\mathrm{cpa}}(q, \tau)=\operatorname{Adv}_{\langle E\rangle,\langle G\rangle}^{\mathrm{cpa}^{\prime}}(q, \tau)
$$

In general, $\mathrm{cpa}^{\prime}$ is weaker than cpa when at least one of two target functions is not invertible. Note that, following the invertibility condition does not exclude all collisions that can not be happened for permutations. For example, if a cpa'-adversary is interacting with $F=\langle G\rangle$ for some keyed permutation $G, M_{i} \neq Y_{j}$ holds true for all $i<j$ with $W_{i} \neq W_{j}$ (in addition to $Y_{i} \neq M_{j}$, which is guaranteed from the invertibility condition). However $M_{i}=Y_{j}$ can happen when (e.g.) $F$ is a URF, as $Y_{j}$ is uniform and independent of $X_{i}$ for all $i<j$.

\subsection{Maurer's Methodology}

Our security proof will be based on a methodology developed by Maurer [19]. Here, we briefly describe it. See Maurer [19] for a more detailed description. Consider a binary random variable $A_{i}$ as a (non-deterministic) function of $i$ input/output pairs (and internal variables) of a keyed function. We denote the event $A_{i}=1$ by $a_{i}$, and denote $A_{i}=0$ by $\overline{a_{i}}$. We assume $a_{i}$ is monotone; i.e., $a_{i}$ never occurs if $\overline{a_{i-1}}$ occurs. For instance, $a_{i}$ is monotone if it indicates that all $i$ outputs are distinct. An infinite sequence $\mathcal{A}=a_{0} a_{1} \ldots$ is called a monotone event sequence (MES). Here, $a_{0}$ is some tautological event (i.e. $A_{0}=1$ with probability 1). Note that $\mathcal{A} \wedge \mathcal{B}=\left(a_{0} \wedge b_{0}\right)\left(a_{1} \wedge b_{1}\right) \ldots$ is an MES if $\mathcal{A}=a_{0} a_{1} \ldots$ and $\mathcal{B}=b_{0} b_{1} \ldots$ are both MESs. For any sequence of random variables, $X_{1}, X_{2}, \ldots$, let $X^{i}$ denote $\left(X_{1}, \ldots, X_{i}\right)$. Let MESs $\mathcal{A}$ and $\mathcal{B}$ be defined for two keyed functions, $F: \mathcal{X} \rightarrow \mathcal{Y}$ and $G: \mathcal{X} \rightarrow \mathcal{Y}$, respectively. Let $X_{i} \in \mathcal{X}$ and $Y_{i} \in \mathcal{Y}$ be the $i$-th input and output. Let $P^{F}$ be the probability space defined by $F$. For example, $P_{Y_{i} \mid X^{i} Y^{i-1}}^{F}\left(y_{i}, x^{i}, y^{i-1}\right)$ means $\operatorname{Pr}\left[Y_{i}=y_{i} \mid X^{i}=x^{i}, Y^{i-1}=y^{i-1}\right]$ where $Y_{j}=F\left(X_{j}\right)$ for $j \geq 1$. If $P_{Y_{i} \mid X^{i} Y^{i-1}}^{F}\left(y_{i}, x^{i}, y^{i-1}\right)=P_{Y_{i} \mid X^{i} Y^{i-1}}^{G}\left(y_{i}, x^{i}, y^{i-1}\right)$ for all possible $\left(x^{i}, y^{i-1}\right)$, then we write $P_{Y_{i} \mid X^{i} Y^{i-1}}^{F}=P_{Y_{i} \mid X^{i} Y^{i-1}}^{G}$ and denote it by $F \equiv G$. Here, note that the definitions of $\mathcal{X}$ and $\mathcal{Y}$, and the set of possible $\left(x^{i}, y^{i-1}\right)$ may depend on the target attack class. Inequalities such as $P_{Y_{i} \mid X^{i} Y^{i-1}}^{F} \leq P_{Y_{i} \mid X^{i} Y^{i-1}}^{G}$ are similarly defined. 
Definition 1. We write $F^{\mathcal{A}} \equiv G^{\mathcal{B}}$ if $P_{Y_{i} a_{i} \mid X^{i} Y^{i-1} a_{i-1}}^{F}=P_{Y_{i} b_{i} \mid X^{i} Y^{i-1} b_{i-1}}^{G}$ hold 2 for all $i \geq 1$. Moreover, we write $F|\mathcal{A} \equiv G| \mathcal{B}$ if $P_{Y_{i} \mid X^{i} Y^{i-1} a_{i}}^{F}=P_{Y_{i} \mid X^{i} Y^{i-1} b_{i}}^{G}$ holds for all $i \geq 1$.

Definition 2. For $M E S \mathcal{A}$ defined for $F, \nu_{\text {atk }}\left(F, \overline{a_{q}}\right)$ denote $\sqrt{3}$ the maximal probability of $\overline{a_{q}}$ for any atk-adversary using $q$ queries (and infinite computational power) that interacts with $F$.

The equivalences defined by Definition 1 are crucial to information-theoretic security proofs. For example, the following theorem holds true.

Theorem 1. (Theorem 1 (i) of [19]) If $F^{\mathcal{A}} \equiv G^{\mathcal{B}}$ or $F \mid \mathcal{A} \equiv G$ holds for an attack class atk, then $\operatorname{Adv}_{F, G}^{\text {atk }}(q) \leq \nu_{\text {atk }}\left(F, \overline{a_{q}}\right)$.

We will use some of Maurer's results including Theorem 11 to make simple and intuitive proofs . For completeness, these results are cited in Appendix $\mathrm{A}$.

\section{Previous Constructions of DBLC}

There are many $O\left(2^{n / 2}\right)$-secure DBLC proposals. Luby and Rackoff proved that the 4-round random Feistel cipher (denoted by $\psi_{4}$ ) is $O\left(2^{n / 2}\right)$-secure. Here, "random" means that each round function is an independent $n$-bit block PRFs. Later, Naor and Reingold [22] proved that the first and last round functions of $\psi_{4}$ need not necessarily be pseudorandom, but only required to be $\epsilon$-almost XOR uniform ( $\epsilon$-AXU) for sufficiently small $\epsilon$. Here, if $H$ is a keyed function being $\epsilon$-AXU, we have $\operatorname{Pr}\left[H(x) \oplus H\left(x^{\prime}\right)=\delta\right] \leq \epsilon$ for any $x \neq x$ and $\delta$. The result of [22] stimulated many related works, e.g., $23,13,26$, to name a few. Above all, what inspired us was another proposal of Naor and Reingold 22, which is so-called NR mode. Basically it is an $m n$-bit block cipher for arbitrarily large $m$, using $n$-bit block cipher, $E$. When $m=2$, NR mode encrypts a plaintext $M \in \Sigma^{2 n}$ as:

$$
C=G_{2}^{-1} \circ \operatorname{ECB}[E] \circ G_{1}(M),
$$

where $\mathrm{ECB}[E]$ is the $2 n$-bit permutation from ECB mode of $E . G_{1}$ and $G_{2}$ are keyed permutations called pairwise independent permutations 22. That is, $\operatorname{Pr}\left[G_{i}(x)=y, G_{i}\left(x^{\prime}\right)=y^{\prime}\right]=1 /\left(2^{n} \cdot\left(2^{n}-1\right)\right)$ for any $x \neq x^{\prime}$ and $y \neq y^{\prime}$, where probability is defined by the distribution of $G_{i}$ 's key for $i=1,2$.

Compared to the vast amount of $O\left(2^{n / 2}\right)$-secure proposals, we have very few schemes achieving better security. A scheme of Aiello and Venkatesan [1] has some beyond-birthday-bound security but not invertible. A proposal of [22] was based on unbalanced Feistel round, where each round function has inputs longer than $n$-bit. The $O\left(2^{n / 2}\right)$-security of $\psi_{6}$ was proved by Patarin [18 and another proof of $\psi_{r}$ for $r \rightarrow \infty$ was given by Maurer and Pietrzak [20, though we omit the details here.

\footnotetext{
${ }^{2}$ As $a_{i}$ denotes $A_{i}=1$, this equality means $P_{Y_{i} A_{i} \mid X^{i} Y^{i-1} A_{i-1}}^{F}\left(y_{i}, 1, x^{i}, y^{i-1}, 1\right)$ equals to $P_{Y_{i} B_{i} \mid X^{i} Y^{i-1} B_{i-1}}^{G}\left(y_{i}, 1, x^{i}, y^{i-1}, 1\right)$ for all $\left(x^{i}, y^{i-1}\right)$ such that both $P_{A_{i-1} X^{i} Y^{i-1}}^{F}\left(1, x^{i}, y^{i-1}\right)$ and $P_{B_{i-1} X^{i} Y^{i-1}}^{G}\left(1, x^{i}, y^{i-1}\right)$ are positive.

3 The original definition does not contain atk; this is for readability.
} 


\section{Building a DBLC with Beyond-Birthday-Bound Security}

\subsection{Extending Naor-Reingold Approach}

Our goal is to build a $O\left(2^{\omega+n / 2}\right)$-secure DBLC, i.e., an $2 n$-bit keyed permutation which is hard to be distinguished from $\mathrm{P}_{2 n}$ against any practical CCA using $q \ll$ $2^{\omega+n / 2}$ queries for some $\omega>0$ (a large $\omega$ indicates a strong security). Our initial question is if we can adopt a Mix-Encrypt-Mix structure similar to Eq. (3). In the following, we provide a novel solution using tweakable block ciphers. The scheme has Mix-Encrypt-Mix structure similar to NR mode, thus we call our scheme Extended Naor-Reingold (ENR) 5 . It has a parameter $m \in\{1, \ldots, n\}$, and we will prove $O\left(2^{(n+m) / 2}\right)$-security.

For convenience, for any random variable $X$, we abbreviate $X_{[1, m]}$ to $\widehat{X}$ (i.e., $\widehat{X}$ is the first $m$-bit of $X)$. If $|X|=m$, we have $\widehat{X}=X$. Let $\widetilde{E}$ be an $(n, m)$-bit tweakable cipher, and let $\widetilde{E}_{L}$ and $\widetilde{E}_{R}$ denote two independently-keyed instances of $\widetilde{E}$. ENR consists of $\widetilde{E}_{L}, \widetilde{E}_{R}$, and a $2 n$-bit keyed permutation, $G$. For plaintext $\left(M_{l}, M_{r}\right) \in \Sigma^{n} \times \Sigma^{n}$ and ciphertext $\left(C_{l}, C_{r}\right) \in \Sigma^{n} \times \Sigma^{n}$, the encryption and decryption of ENR are defined as Fig.11.

Algorithm 4.1: $\operatorname{ENR}[G, \widetilde{E}]\left(M_{l}, M_{r}\right)$

$$
\begin{aligned}
& (S, T) \leftarrow G\left(M_{l}, M_{r}\right) \\
& U \leftarrow \widetilde{E}_{L}(S, \widehat{T}), \quad V \leftarrow \widetilde{E}_{R}(T, \widehat{U}) \\
& \left(C_{l}, C_{r}\right) \leftarrow G_{\text {rev }}^{-1}(U, V) \\
& \text { return }\left(\left(C_{l}, C_{r}\right)\right)
\end{aligned}
$$

Algorithm 4.2: $\operatorname{ENR}[G, \widetilde{E}]^{-1}\left(C_{l}, C_{r}\right)$

$$
\begin{aligned}
& (U, V) \leftarrow G_{\text {rev }}\left(C_{l}, C_{r}\right) \\
& T \leftarrow \widetilde{E}_{R}^{-1}(V, \widehat{U}), \quad S \leftarrow \widetilde{E}_{L}^{-1}(U, \widehat{T}) \\
& \left(M_{l}, M_{r}\right) \leftarrow G^{-1}(S, T) \\
& \text { return }\left(\left(M_{l}, M_{r}\right)\right)
\end{aligned}
$$

Fig. 1. Encryption (left) and decryption (right) procedures of ENR

Here, $G_{\text {rev }}$ denotes the mirrored image of $G$, i.e., $G_{\text {rev }}(x)=\operatorname{rev}(G(\operatorname{rev}(x)))$ with $\operatorname{rev}\left(x_{1}, \ldots, x_{2 n}\right)=\left(x_{2 n}, \ldots, x_{1}\right)$. We assume $G_{\text {rev }}$ and $G$ use the same key. Basically, we can prove the security of ENR for a more general setting where the second mixing layer is not restricted to $G_{\mathrm{rev}}$. We here focus on the use of $G_{\mathrm{rev}}$ because it allows us to reuse the key and implementation of $G$.

\subsection{Security Proof of ENR}

To prove the security of $\operatorname{ENR}[G, \widetilde{E}]$, we first introduce a condition for $G$.

\footnotetext{
${ }^{4}$ Naor and Reingold's unbalanced Feistel cipher is based on Mix-Encrypt-Mix structure and achieves $O\left(2^{\omega+n / 2}\right)$-security. However, as it uses PRFs with input longer than $n$-bit, it is not comparable to ours. Moreover, an important difference is that the number of round of their scheme is depending on the security parameter (for higher security more rounds are needed), while that of ours is constant.

${ }^{5}$ If our scheme is realized with non-tweakable permutation (by setting $m=0$ ), it will be very close to NR mode.
} 
Definition 3. Let $G$ be a $2 n$-bit keyed permutation. Let $m \in\{1, \ldots, n\}$ be $a$ parameter. If $G$ is $(\epsilon, \gamma, \rho)$-almost uniform $((\epsilon, \gamma, \rho)$-AU), we have

$$
\begin{aligned}
& \operatorname{Pr}\left[G(x)_{[1, n+m]}=G\left(x^{\prime}\right)_{[1, n+m]}\right] \leq \epsilon, \text { and } \\
& \operatorname{Pr}\left[G(x)_{[n+1,2 n]}=G\left(x^{\prime}\right)_{[n+1,2 n]}\right] \leq \gamma, \text { and } \\
& \operatorname{Pr}\left[G(x)_{[n+1, n+m]}=G\left(x^{\prime}\right)_{[n+1, n+m]}\right] \leq \rho, \text { for any distinct } x, x^{\prime} \in \Sigma^{2 n} .
\end{aligned}
$$

A $2 n$-bit pairwise independent permutation is $\left(2^{-(n+m)}, 2^{-n}, 2^{-m}\right)$-AU. Even a more efficient construction is possible by using Feistel permutation (see Corollaries 1 and 2). The security proof of general ENR is as follows.

Theorem 2. If $G$ is $(\epsilon, \gamma, \rho)-A U$ for $m \in\{1, \ldots, n\}$ and $\widetilde{E}$ is an $(n, m)$-bit tweakable cipher, we have

$$
\begin{aligned}
& \operatorname{Adv}_{\operatorname{ENR}[G, \widetilde{E}]}^{\operatorname{sprp}}(q, \tau) \\
& \leq 2 \operatorname{Adv}_{\widetilde{E}}^{\widetilde{\operatorname{sprp}}}(q, \tau+O(q))+q^{2}\left(3 \epsilon+\frac{2 \gamma}{2^{m}}+\frac{\rho}{2^{n}}+\max \left\{\frac{\gamma}{2^{m}}, \frac{\rho}{2^{n}}\right\}+\frac{1}{2^{n+m}}\right) .
\end{aligned}
$$

We also provide two instantiations of ENR with Feistel-based implementations of $G$.

Corollary 1. Let $m=n$ and $\psi[H]$ be a balanced $2 n$-bit (left-to-right, see the left of Fig. (2) Feistel using a round function $H: \Sigma^{n} \rightarrow \Sigma^{n}$. $H$ is defined as $H(x)=K \cdot x$, where multiplication is defined over $\mathrm{GF}\left(2^{n}\right)$ and key $K$ is uniformly random over $\mathrm{GF}\left(2^{n}\right)$. Then we have

$$
\operatorname{Adv}_{\operatorname{ENR}[\psi[H], \widetilde{E}]}^{\operatorname{sprp}}(q, \tau) \leq 2 \operatorname{Adv}_{\widetilde{E}}^{\widetilde{\operatorname{sprp}}}(q, \tau+O(q))+\frac{5 q^{2}}{2^{2 n}} .
$$

Proof. When $m=n$, every $2 n$-bit keyed permutation is $(0, \gamma, \rho)$-AU for some $\gamma=\rho$. The probability of $\psi[H](x)_{[n+1, \ldots, 2 n]}=\psi[H]\left(x^{\prime}\right)_{[n+1, \ldots, 2 n]}$ is at most $\gamma$ for any $x \neq x^{\prime}$, if $H$ is $\gamma$-AXU. Here, our $H$ is $2^{-n}$-AXU, thus $\psi[H]$ is $\left(0,2^{-n}, 2^{-n}\right)$ AU. Combining this fact and Theorem 2 proves the corollary.

Corollary 2. Let $m<n$, and $K_{1}, K_{2}$, and $K_{3}$ be independent and uniform over $\mathrm{GF}\left(2^{n}\right)$ (represented as $n$-bit values). We define $H_{1}: \Sigma^{n} \rightarrow \Sigma^{n}$ as $H_{1}(x)=$ $K_{1} \cdot x$, and define $H_{2}: \Sigma^{n-m} \rightarrow \Sigma^{n+m}$ as $H_{2}(x)=\left(K_{2} \cdot \dot{x} \| K_{3} \cdot \dot{x}\right)_{[1, \ldots, n+m]}$, where $\dot{x}=x \| 0^{m}$. Then,

$$
\operatorname{Adv}_{\operatorname{ENR}\left[\psi\left[H_{1}, H_{2}\right], \widetilde{E}\right]}^{\operatorname{sprp}}(q, \tau) \leq 2 \operatorname{Adv}_{\widetilde{E}}^{\widetilde{\operatorname{sprp}}}(q, \tau+O(q))+q^{2}\left(\frac{8}{2^{n+m}}+\frac{2}{2^{2 n}}\right),
$$

where $\psi\left[H_{1}, H_{2}\right]$ is a 2-round Feistel permutation with $i$-th round function $H_{i}$ (the first round is balanced and the second is unbalanced, see the right of Fig.2).

Proof. We show that $\psi\left[H_{1}, H_{2}\right]$ is $(\epsilon, \gamma, \rho)$-AU with $\epsilon=2^{-(n+m)}, \gamma=2^{-n}$, and $\rho=2^{-n}+2^{-m}$. The proofs for $\epsilon$ and $\gamma$ are easy, as $H_{2}$ is $2^{-(n+m)}-\mathrm{AXU}$ and $H_{1}$ is $2^{-n}$-AXU. To prove $\rho$, let $\mathcal{E}_{1}$ denote the collision event on $\psi\left[H_{1}\right](x)_{[1, \ldots, n]}$ and 
let $\mathcal{E}_{2}$ denote the collision event on $\psi\left[H_{1}, H_{2}\right](x)_{[n+1, \ldots, n+m]}$. Here, $\rho$ is obtained by bounding $\operatorname{Pr}\left(\mathcal{E}_{2}\right)$ for any two distinct inputs to $\psi\left[H_{1}, H_{2}\right]$, which is as follows. $\operatorname{Pr}\left(\mathcal{E}_{2}\right)=\operatorname{Pr}\left(\mathcal{E}_{1}\right) \cdot \operatorname{Pr}\left(\mathcal{E}_{2} \mid \mathcal{E}_{1}\right)+\operatorname{Pr}\left(\overline{\mathcal{E}_{1}}\right) \cdot \operatorname{Pr}\left(\mathcal{E}_{2} \mid \overline{\mathcal{E}_{1}}\right) \leq \operatorname{Pr}\left(\mathcal{E}_{1}\right)+\operatorname{Pr}\left(\mathcal{E}_{2} \mid \overline{\mathcal{E}_{1}}\right) \leq 2^{-n}+2^{-m}$, where the last inequality follows from that $H_{1}$ is $2^{-n}$-AXU and $H_{2}(x)_{[n+1, \ldots, n+m]}$ is $2^{-m}$-AXU. Combining this observation and Theorem 2 , the proof is completed.
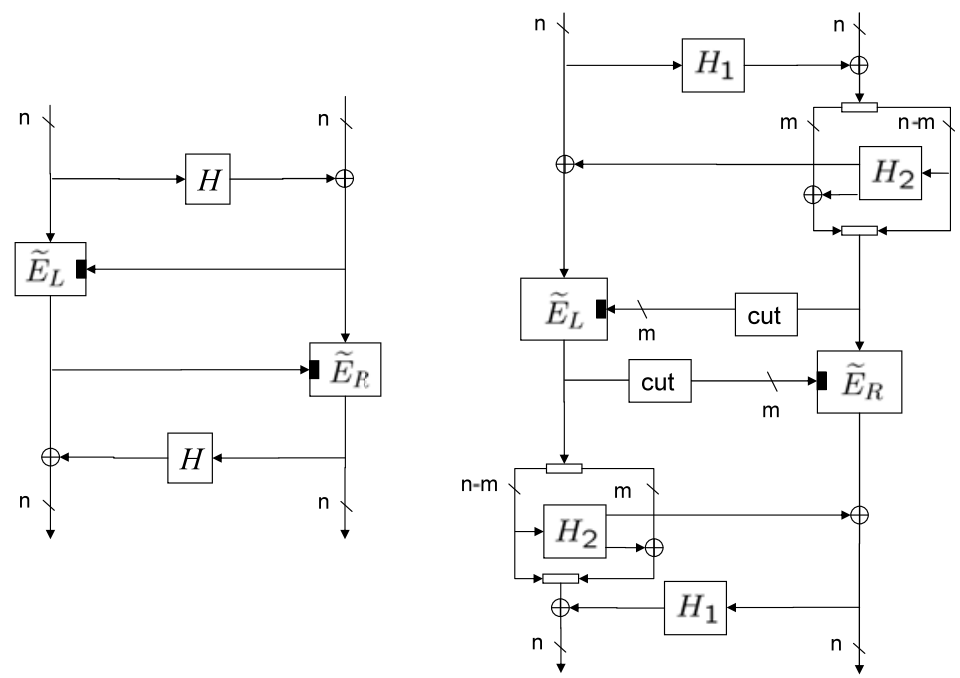

Fig. 2. Encryption of ENR. Left: the case $m=n$. Right: the case $m<n$.

\subsection{Proof of Theorem 2}

Setup. Let us abbreviate $\operatorname{ENR}\left[G, \widetilde{\mathrm{P}}_{n, m}\right]$ to $\operatorname{ENR}^{*}$, where $G$ is $(\epsilon, \gamma, \rho)$-AU. We only present the information-theoretic part, that is, the indistinguishability of ENR $^{*}$ from $\mathrm{P}_{2 n}$ against any computationally unbounded cca-adversary. The computational part is easy from the standard technique (see e.g., [2]). For convenience, we introduce some notations. For any $F: \mathcal{M} \times \Sigma \rightarrow \mathcal{M}$ with a set $\mathcal{M}$, $F[i]: \mathcal{M} \rightarrow \mathcal{M}$ is defined as $F[i](x)=F(x, i)$ for $i \in \Sigma$. For $F: \Sigma^{2 n} \times \Sigma \rightarrow \Sigma^{2 n}$, we define $\mathbb{G} F: \Sigma^{2 n} \times \Sigma \rightarrow \Sigma^{2 n}$ as

$$
\mathbb{G} F(x, 0)=G_{\mathrm{rev}}^{-1} \circ F[0] \circ G(x), \quad \text { and } \mathbb{G} F(x, 1)=G^{-1} \circ F[1] \circ G_{\mathrm{rev}}(x) .
$$

Then, DR : $\Sigma^{2 n} \times \Sigma \rightarrow \Sigma^{2 n}$ is defined as

$$
\begin{aligned}
& \operatorname{DR}\left(\left(x_{l}, x_{r}\right), 0\right)=\left(U, \mathrm{R}_{R}\left(x_{r}, \widehat{U}, 0\right)\right), \text { where } U=\mathrm{R}_{L}\left(x_{l}, \widehat{x_{r}}, 0\right), \\
& \operatorname{DR}\left(\left(x_{l}, x_{r}\right), 1\right)=\left(\mathrm{R}_{R}\left(x_{l}, \widehat{T}, 1\right), T\right), \text { where } T=\mathrm{R}_{L}\left(x_{r}, \widehat{x_{l}}, 1\right),
\end{aligned}
$$


using two independent URFs, $\mathrm{R}_{L}, \mathrm{R}_{R}: \Sigma^{n} \times \Sigma^{m} \times \Sigma \rightarrow \Sigma^{n}$. Let $\widetilde{\mathrm{P}}_{L}$ and $\widetilde{\mathrm{P}}_{R}$ denote two independent instances of $\widetilde{\mathrm{P}}_{n, m}$. Using them, we also define DP : $\Sigma^{2 n} \times \Sigma \rightarrow \Sigma^{2 n}$ in the same way as DR but $\mathrm{R}_{R}$ and $\mathrm{R}_{L}$ are substituted with $\left\langle\widetilde{\mathrm{P}}_{R}\right\rangle$ and $\left\langle\widetilde{\mathrm{P}}_{L}\right\rangle$, respectively. Here, note that $\mathbb{G D P}$ is equivalent to $\left\langle\mathrm{ENR}^{*}\right\rangle$.

The proof outline is as follows. We analyze cpa'-advantage between GDP and $\left\langle\mathrm{P}_{2 n}\right\rangle$, which corresponds to what we want from Eq. (2). Then, using the triangle inequality, we move as $\mathbb{G D P} \Rightarrow \mathbb{G D R} \Rightarrow \mathrm{R}_{2 n+1,2 n} \Rightarrow\left\langle\mathrm{P}_{2 n}\right\rangle$, that is, we evaluate the maximum cpa'-advantages for the game with $\mathbb{G D P}$ and $\mathbb{G D R}$ (Game 1), and the game with GDR and $\mathrm{R}_{2 n+1,2 n}$ (Game 2), and the game with $\mathrm{R}_{2 n+1,2 n}$ and $\left\langle\mathrm{P}_{2 n}\right\rangle$ (Game 3). Formally, we have

$$
\begin{aligned}
& \operatorname{Adv}_{\mathrm{ENR}^{*}}^{\operatorname{sprp}}(q)=\operatorname{Adv}_{\mathrm{ENR}^{*}, \mathrm{P}_{2 n}}^{\mathrm{cca}}(q)=\operatorname{Adv}_{\left\langle\mathrm{ENR}^{*}\right\rangle,\left\langle\mathrm{P}_{2 n}\right\rangle}^{\mathrm{cpa}^{\prime}}=\operatorname{Adv}_{\mathbb{G D P},\left\langle\mathrm{P}_{2 n}\right\rangle}^{c \mathrm{cpa}^{\prime}} \\
& \leq \operatorname{Adv}_{\mathbb{G D P}, \mathbb{G D R}}^{\mathrm{cpa}^{\prime}}(q)+\operatorname{Adv}_{\mathbb{G D R}, \mathrm{R}_{2 n+1,2 n}}^{\mathrm{cpa}{ }^{\prime}}(q)+\operatorname{Adv}_{\mathrm{R}_{2 n+1,2 n},\left\langle\mathrm{P}_{2 n}\right\rangle}^{\mathrm{ppa}^{\prime}}(q) .
\end{aligned}
$$

Analysis of Game 3. By extending the well-known PRP-PRF switching lemma (e.g., Lemma 1 of [4]), we easily get

$$
\operatorname{Adv}_{\mathrm{R}_{2 n+1,2 n},\left\langle\mathrm{P}_{2 n}\right\rangle}^{\mathrm{ppa}^{\prime}}(q) \leq\left(\begin{array}{l}
q \\
2
\end{array}\right) \cdot \frac{1}{2^{2 n}} .
$$

Analysis of Game 2. We first observe that

$$
\mathbb{G R}_{2 n+1,2 n} \equiv \mathrm{R}_{2 n+1,2 n} \text {, and thus } \operatorname{Adv}_{\mathbb{G D R}, \mathrm{R}_{2 n+1,2 n}}^{\mathrm{cpa}^{\prime}}(q)=\operatorname{Adv}_{\mathbb{G D R}, \mathbb{G R} \mathrm{R}_{2 n+1,2 n}}^{\mathrm{cpa}^{\prime}}(q) \text {, }
$$

since pre- and post-processing added by $\mathbb{G}$ are permutations. We consider an adversary, $\mathcal{D}$, accessing to $F$ which is $\mathrm{DR}$ or $\mathrm{R}_{2 n+1,2 n}$. For each time period $i=1, \ldots, q, \mathcal{D}$ can choose whether $F[0]$ or $F[1]$ is queried. This information is denoted by $W_{i} \in \Sigma$, and if $W_{i}=0$, the input to $F[0]$ is denoted by $\left(S E_{i}, T E_{i}\right) \in$ $\Sigma^{n} \times \Sigma^{n}$ (if $W_{i}=1,\left(S E_{i}, T E_{i}\right)$ is undefined), and the corresponding output is denoted by $\left(U E_{i}, V E_{i}\right) \in \Sigma^{n} \times \Sigma^{n}$. Similarly, if $W_{i}=1$, the input to $F[1]$ and the output from $F[1]$ are denoted by $\left(U D_{i}, V D_{i}\right)$ and $\left(S D_{i}, T D_{i}\right)$, respectively (see Fig.3). These notations will also be used for adversaries accessing to $\mathbb{G} F$. We define an $\operatorname{MES} \mathcal{E}=e_{0} e_{1} \ldots$, where $e_{q}$ denotes the event that

$$
\begin{aligned}
& \left(S E_{i}, \widehat{T E}_{i}\right) \neq\left(S E_{j}, \widehat{T E}_{j}\right) \text { and }\left(\widehat{U E}_{i}, T E_{i}\right) \neq\left(\widehat{U E}_{j}, V E_{j}\right), \text { and } \\
& \left(U D_{i}, \widehat{T D}_{i}\right) \neq\left(U D_{j}, \widehat{T D}_{j}\right) \text { and }\left(\widehat{U D}_{i}, V D_{i}\right) \neq\left(\widehat{U D}_{j}, V D_{j}\right),
\end{aligned}
$$

holds for all possible $i \neq j, i, j \in\{1, \ldots, q\}$, e.g., Eq. (10) for $i \neq j$ with $W_{i}=$ $W_{j}=0$. Then, we obtain the following equivalence. Its proof is in Appendix B.

$$
\mathrm{DR}^{\mathcal{E}} \equiv \mathrm{R}_{2 n+1,2 n}^{\mathcal{E}}
$$

From Eq. (12) and Lemma 2, we have

$$
\mathbb{G D R}^{\mathcal{E}} \equiv \mathbb{G R}_{2 n+1,2 n}^{\mathcal{E}}
$$

Using Eqs. (9) and (13) and Theorem 1, we obtain

$$
\operatorname{Adv}_{\mathbb{G D R}, \mathrm{R}_{2 n+1,2 n}}^{\mathrm{cpa}^{\prime}}(q)=\operatorname{Adv}_{\mathbb{G D R}, \mathbb{G R} R_{2 n+1,2 n}}^{\mathrm{cpa}^{\prime}}(q) \leq \nu_{\mathrm{cpa}^{\prime}}\left(\mathbb{G R}_{2 n+1,2 n}, \overline{e_{q}}\right)
$$


We leave the analysis of the last term of Eq. (14) for now.

Analysis of Game 1. We consider the indistinguishability between $\left\langle\widetilde{\mathrm{P}}_{n, m}\right\rangle$ and $\mathrm{R}_{n+m+1, n}$. We first focus on the input/output collision for the same tweak value. More precisely, let $\left(X_{i}, T_{i}, W_{i}\right) \in \Sigma^{n} \times \Sigma^{m} \times \Sigma$ denote the $i$-th input to $\left\langle\widetilde{\mathrm{P}}_{n, m}\right\rangle$ or $\mathrm{R}_{n+m+1, n}$, and let $Y_{i} \in \Sigma^{n}$ be the $i$-th output. For $b \in \Sigma$ and $t \in \Sigma^{m}$, let $\mathcal{X}_{b}^{t}=\left\{X_{i}: i \in\{1, \ldots, q\}, T_{i}=t, W_{i}=b\right\}$ and $\mathcal{Y}_{b}^{t}=\left\{Y_{i}: i \in\{1, \ldots, q\}, T_{i}=\right.$ $\left.t, W_{i}=b\right\}$. Then, $a_{q}$ denotes the event that

$$
\left[\mathcal{X}_{0}^{t} \cap \mathcal{Y}_{1}^{t}=\emptyset\right] \wedge\left[\mathcal{X}_{1}^{t} \cap \mathcal{Y}_{0}^{t}=\emptyset\right] \text { for all } t \in \Sigma^{m} .
$$

The corresponding MES, $\mathcal{A}=a_{0} a_{1} \ldots$, is called the generalized collision-freeness (GCF). Then, we have

$$
\left\langle\widetilde{\mathrm{P}}_{n, m}\right\rangle^{\mathcal{A} \wedge \mathcal{C}} \equiv \mathrm{R}_{n+m+1, n}^{\mathcal{A}}, \text { for some } \operatorname{MES} \mathcal{C} .
$$

The proof of Eq. (15) is written in Appendix C. As mentioned, if we substitute $\mathrm{R}_{L}$ and $\mathrm{R}_{R}$ with $\left\langle\widetilde{\mathrm{P}}_{L}\right\rangle$ and $\left\langle\widetilde{\mathrm{P}}_{R}\right\rangle$, we will obtain DP. Thus, from Eq. (15), we get

$$
\mathrm{DP}^{\mathcal{A} \mathcal{L} \wedge \mathcal{C} \mathcal{L} \wedge \mathcal{A} \mathcal{R} \wedge \mathcal{C} \mathcal{R}} \equiv \mathrm{DR}^{\mathcal{A L} \wedge \mathcal{A R}} \text {, and } \mathbb{G D P}^{\mathcal{A} \mathcal{L} \wedge \mathcal{C} \mathcal{L} \wedge \mathcal{A R} \wedge \mathcal{C} \mathcal{R}} \equiv \mathbb{G D R}^{\mathcal{A L} \wedge \mathcal{A R}}
$$

where $\mathcal{A L}=a l_{0} a l_{1} \ldots$ denotes the GCF for $\left\langle\widetilde{\mathrm{P}}_{L}\right\rangle$ or $\mathrm{R}_{L}$, and $\mathcal{A} \mathcal{R}=a r_{0} a r_{1} \ldots$ denotes the GCF for $\left\langle\widetilde{\mathrm{P}}_{R}\right\rangle$ or $\mathrm{R}_{R}$, and $\mathcal{C} \mathcal{L}$ and $\mathcal{C} \mathcal{R}$ are some MESs (implied by Eq. (15) ). The second equivalence follows from Lemma 2 Thus, using Theorem 1 we obtain

$$
\operatorname{Adv}_{\mathbb{G D P}, \mathbb{G D R}}^{\mathrm{cpa}^{\prime}}(q) \leq \nu_{\mathrm{cpa}^{\prime}}\left(\mathbb{G D R}, \overline{a l_{q} \wedge a r_{q}}\right)
$$

For DR and $\mathrm{R}_{2 n+1,2 n}$, the occurrence of $a l_{q} \wedge a r_{q}$ can be completely determined by the $q$ inputs and outputs. From this fact and Lemma 5, we can adjoin $\mathcal{A L} \wedge \mathcal{A} \mathcal{R}$ to the both sides of Eq. (12) and obtain

$$
\mathrm{DR}^{\mathcal{E} \wedge \mathcal{A} \mathcal{L} \wedge \mathcal{A R}} \equiv \mathrm{R}_{2 n+1,2 n}^{\mathcal{E} \wedge \mathcal{A} \mathcal{L} \wedge \mathcal{A} \mathcal{R}}
$$

Moreover, it is easy to see that $\mathcal{E} \wedge \mathcal{A L} \wedge \mathcal{A R} \equiv \mathcal{A L} \wedge \mathcal{A R}$ holds for DR and $\mathrm{R}_{2 n+1,2 n}$. Combining this observation, Eq. (18), and Lemmas 2 and 3 , we have

$$
\begin{aligned}
& \mathbb{G D R}^{\mathcal{A L} \wedge \mathcal{A R}} \equiv \mathbb{G R}_{2 n+1,2 n}^{\mathcal{A L} \wedge \mathcal{A R}}, \text { and } \\
& \operatorname{Adv}_{\mathbb{G D P}, \mathbb{G D R}}^{\mathrm{cpa}^{\prime}}(q) \leq \nu_{\mathrm{cpa}^{\prime}}\left(\mathbb{G D R}, \overline{a l_{q} \wedge a r_{q}}\right)=\nu_{\mathrm{cpa}^{\prime}}\left(\mathbb{G R}_{2 n+1,2 n}, \overline{a l_{q} \wedge a r_{q}}\right) .
\end{aligned}
$$

Collision Probability analysis. Combining Eqs. (7), (8), (14), and (19), and Lemma 4, we have

$$
\operatorname{Adv}_{\operatorname{ENR}^{*}}^{\operatorname{sprp}}(q) \leq\left(\begin{array}{l}
q \\
2
\end{array}\right) \frac{1}{2^{2 n}}+\sum_{\mathbf{e v}=\overline{e_{q}}, \overline{a l_{q}}, \overline{a r_{q}}} \nu_{\mathrm{cpa}^{\prime}}\left(\mathbb{G R}_{2 n+1,2 n}, \mathbf{e v}\right)
$$

We need to bound $\nu_{\mathrm{cpa}^{\prime}}$ terms of Eq. (20). First, the maximum probabilities of $\overline{a l_{q}}$ and $\overline{a r_{q}}$ (under $\mathbb{G R}_{2 n+1,2 n}$ ) are the same because of the symmetry from Eq. (4) 

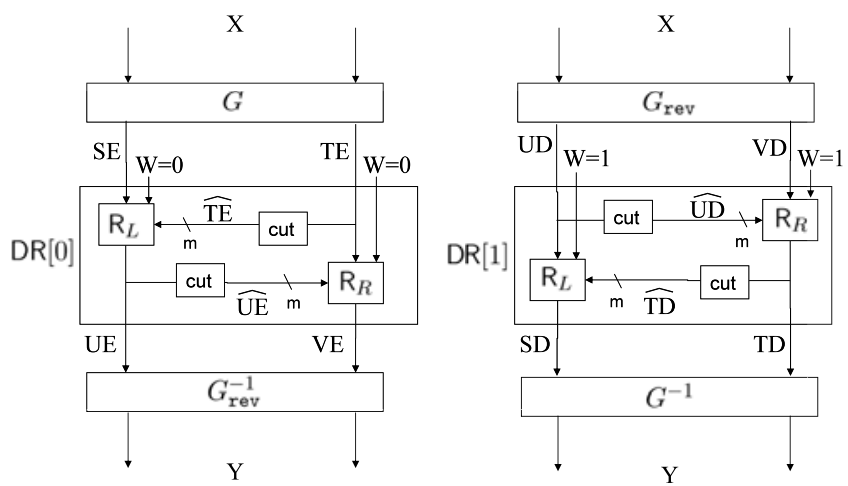

Fig. 3. GDR function

and that $G_{\text {rev }}$ is a mirrored image of $G$. Thus we only need to evaluate the maximum probabilities of $\overline{a l_{q}}$ and $\overline{e_{q}}$.

As shown by Eqs. (10) and (11), $\overline{e_{q}}$ consists of collision events such as

$$
\begin{aligned}
& \operatorname{type}\left[\mathbf{e}_{1}\right]:\left(S E_{i}, \widehat{T E}_{i}\right)=\left(S E_{j}, \widehat{T E}_{j}\right), \quad \operatorname{type}\left[\mathbf{e}_{2}\right]:\left(\widehat{U E}_{i}, T E_{i}\right)=\left(\widehat{U E}_{j}, T E_{j}\right) \\
& \operatorname{type}\left[\mathbf{e}_{3}\right]:\left(\widehat{U D}_{i}, V D_{i}\right)=\left(\widehat{U D}_{j}, V D_{j}\right), \quad \operatorname{type}\left[\mathbf{e}_{4}\right]:\left(U D_{i}, \widehat{T D}_{i}\right)=\left(U D_{j}, \widehat{T D}_{j}\right)
\end{aligned}
$$

for all possible $i \neq j, i, j \in\{1, \ldots, q\}$. Moreover, $\overline{a l_{q}}$ consists of collision events such as

$$
\begin{array}{ll}
\operatorname{type}\left[\mathbf{a}_{1}\right]:\left(S E_{i}, \widehat{T E}_{i}\right)=\left(S E_{j}, \widehat{T E}_{j}\right), & \operatorname{type}\left[\mathbf{a}_{2}\right]:\left(U E_{i}, \widehat{T E}_{i}\right)=\left(U E_{j}, \widehat{T E}_{j}\right) \\
\operatorname{type}\left[\mathbf{a}_{3}\right]:\left(U D_{i}, \widehat{T D}_{i}\right)=\left(U D_{j}, \widehat{T D}_{j}\right), \quad \operatorname{type}\left[\mathbf{a}_{4}\right]:\left(S D_{i}, \widehat{T D}_{i}\right)=\left(S D_{j}, \widehat{T D}_{j}\right) \\
\operatorname{type}\left[\mathbf{a}_{5}\right]:\left(S E_{i}, \widehat{T E}_{i}\right)=\left(S D_{j}, \widehat{T D}_{j}\right), & \operatorname{type}\left[\mathbf{a}_{6}\right]:\left(U E_{i}, \widehat{T E}_{i}\right)=\left(U D_{j}, \widehat{T D}_{j}\right) .
\end{array}
$$

Note that type $\left[\mathbf{a}_{1}\right]$ and type $\left[\mathbf{a}_{3}\right]$ are the same as type $\left[\mathbf{e}_{1}\right]$ and type $\left[\mathbf{e}_{4}\right]$, respectively. Let $\operatorname{Pr}[\mathbf{x}]$ be the maximum probability of type[x]-collision for $\mathbf{x} \in$ $\left\{\mathbf{e}_{1}, \ldots, \mathbf{e}_{4}, \mathbf{a}_{1}, \ldots, \mathbf{a}_{6}\right\}$ under $\mathbb{G R}_{2 n+1,2 n}$, where the maximum is taken for all $q$-cpa' (possibly adaptive) adversaries and for all $i, j \in\{1, \ldots, q\}$ with $i \neq j$. Using the union bound and the symmetry of $\mathbb{G R}_{2 n+1,2 n}[0]$ and $\mathbb{G R}_{2 n+1,2 n}[1]$, the R.H.S. of Eq. (20) is at most

$$
\left(\begin{array}{l}
q \\
2
\end{array}\right)\left(\frac{1}{2^{2 n}}+\sum_{\mathrm{i}=1, \ldots 4} \operatorname{Pr}\left[\mathbf{e}_{\mathrm{i}}\right]+2 \sum_{\mathrm{j}=1, \ldots 6} \operatorname{Pr}\left[\mathbf{a}_{\mathrm{j}}\right]\right) .
$$

From Eq. (9), the adversary's choice must be independent of (the key of) $G$ and $G_{\text {rev }}$. With this fact, each collision probability of Eq. (21) can be easily bounded for any cpa'-adversary if $G$ is $(\epsilon, \gamma, \rho)$-AU 6 . The full description of our analysis

\footnotetext{
${ }^{6}$ Note that if $G$ is $(\epsilon, \gamma, \rho)$-AU, the mirrored image of $G_{\mathrm{rev}}$ is also $(\epsilon, \gamma, \rho)$-AU.
} 
is rather long7, thus we here describe some typical examples. Other collision probabilities can be analyzed in a similar way. Let $X E_{i}$ and $Y E_{i}$ be the $i$-th $2 n$-bit input and output of $\mathbb{G R}_{2 n+1,2 n}$ with $W_{i}=0$ (they are undefined for $i$ satisfying $\left.W_{i}=1\right)$. Similarly, $X D_{i}$ and $Y D_{i}$ denote the $i$-th input and output with $W_{i}=1$.

- type $\left[\mathbf{e}_{1}\right]$. Here, a collision means $G\left(X E_{i}\right)_{[1, \ldots, n+m]}=G\left(X E_{j}\right)_{[1, \ldots, n+m]}$ for $X E_{i} \neq X E_{j}$. Moreover, $X E_{i}$ and $X E_{j}$ are independent of $G$ 's key. Thus we have $\operatorname{Pr}\left[\mathbf{e}_{1}\right] \leq \epsilon$ as $G$ is assumed to be $(\epsilon, \gamma, \rho)$-AU.

- type $\left[\mathbf{e}_{2}\right]$. Without loss of generality, we assume $i<j$. The probability of $T E_{i}=T E_{j}$ is at most $\gamma$, as $G$ is $(\epsilon, \gamma, \rho)$-AU. Since $G$ is invertible, the inputs to $\mathrm{R}_{2 n+1,2 n}[0]$ are always distinct. This implies that $\widehat{U E}_{j}$ is independent of previous variables (including $\widehat{U E}_{i}, T E_{i}$ and $T E_{j}$ ) and uniform, even conditioned by the event $T E_{i}=T E_{j}$. Thus we get

$$
\operatorname{Pr}\left[\mathbf{e}_{2}\right]=\max _{i \neq j} \operatorname{Pr}\left[\widehat{U E}_{i}=\widehat{U E}_{j} \mid T E_{i}=T E_{j}\right] \cdot \operatorname{Pr}\left[T E_{i}=T E_{j}\right] \leq 2^{-m} \gamma
$$

- type $\left[\mathbf{a}_{5}\right]$. When $i<j,\left(S D_{j}, \widehat{T D}_{j}\right)$ is uniform and independent of $\left(S E_{i}, \widehat{T E}_{i}\right)$, thus collision probability is exactly $2^{-(n+m)}$. When $j<i, X E_{i} \neq Y D_{j}$ must hold as we consider cpa'-adversary (i.e., $X E_{i}=Y D_{j}$ for $j<i$ means an intentional invertibility check). Hence

$$
\begin{aligned}
& \operatorname{Pr}\left[\left(S E_{i}, \widehat{T E}_{i}\right)=\left(S D_{j}, \widehat{T D}_{j}\right)\right] \\
& =\operatorname{Pr}\left[G\left(X E_{i}\right)_{[1, \ldots, n+m]}=G\left(Y D_{j}\right)_{[1, \ldots, n+m]}\right] \leq \epsilon .
\end{aligned}
$$

Thus we have $\operatorname{Pr}\left[\mathbf{a}_{5}\right] \leq \max \left\{2^{-(n+m)}, \epsilon\right\}$. Here, $\epsilon \geq 2^{-(n+m)}$ as it is the collision probability over $(n+m)$ bits.

In summary, we obtain all maximum collision probabilities:

$$
\begin{aligned}
& \operatorname{Pr}\left[\mathbf{e}_{1}\right] \leq \epsilon, \quad \operatorname{Pr}\left[\mathbf{e}_{2}\right] \leq 2^{-m} \gamma, \quad \operatorname{Pr}\left[\mathbf{e}_{3}\right] \leq \epsilon, \quad \operatorname{Pr}\left[\mathbf{e}_{4}\right] \leq 2^{-m} \gamma, \\
& \operatorname{Pr}\left[\mathbf{a}_{1}\right] \leq \epsilon, \quad \operatorname{Pr}\left[\mathbf{a}_{2}\right] \leq 2^{-n} \rho, \quad \operatorname{Pr}\left[\mathbf{a}_{3}\right] \leq 2^{-m} \gamma, \\
& \operatorname{Pr}\left[\mathbf{a}_{4}\right] \leq 2^{-(n+m)}, \quad \operatorname{Pr}\left[\mathbf{a}_{5}\right] \leq \epsilon, \quad \operatorname{Pr}\left[\mathbf{a}_{6}\right] \leq \max \left\{2^{-m} \gamma, 2^{-n} \rho\right\} .
\end{aligned}
$$

Combining Eqs. (24) and (21) proves the theorem.

\subsection{PRP and PRF Versions of ENR}

Although our primary target is a DBLC secure against CCA, a slight simplification of our proposal yields a CPA-secure variant of ENR. It saves some operations from the original ENR at the cost of a weaker attack class.

Definition 4. The simplified ENR (sENR) is defined as ENR with $G_{\text {rev }}$ being omitted (or, substituted with the identity function).

\footnotetext{
7 This is mainly because we have to think of the cases when the adversary's choice is
} adaptive, even though it is independent of $G$. 
Corollary 3. Let $G$ be $(\epsilon, \gamma, \rho)-A U$. Then, the cpa-security of $\operatorname{sENR}[G, \widetilde{E}]$ is:

$$
\operatorname{Adv}_{\operatorname{sENR}[G, \widetilde{E}]}^{\operatorname{prp}}(q, \tau) \leq 2 \operatorname{Adv}_{\widetilde{E}}^{\widetilde{p r p}}(q, \tau+O(q))+q^{2}\left(\epsilon+\frac{\gamma}{2^{m}}+\frac{\rho}{2^{n+1}}+\frac{1}{2^{n+m}}\right) .
$$

The proof is similar to that of Theorem 2 thus is omitted here. The reason why $G_{\text {rev }}$ can be omitted is that, we do not have to consider some bad events (e.g., the collision of $(\widehat{U D}, V D))$ that have to be avoided by $G_{\text {rev }}$ when decryption query is possible. Moreover, by truncating the rightmost $n$-bit output, we obtain a PRF : $\Sigma^{2 n} \rightarrow \Sigma^{n}$ which is $O\left(2^{(n+m) / 2}\right)$-secure for any $m=1, \ldots, n$ (the proof is trivial from Corollary 3). We emphasize that ENR, and the simplified ENR, and its truncated-output version are optimally efficient, for they need exactly $c$ calls of $\widetilde{E}$ when the output is $c n$ bits, for $c=1,2$.

\section{A Simple Construction of Tweakable Block Cipher with Beyond-Birthday-Bound Security}

Our proposal requires a tweakable block cipher with beyond-birthday-bound security. Then, one may naturally ask how to realize it. A straightforward approach is building from scratch, e.g., Mercy [9] and HPC [8]. Recent studies [10] 21] demonstrated that adding a tweak to some internal variables of a (generalized) Feistel cipher could yield a secure tweakable block cipher. This technique, called direct tweaking, may well be applied to a concrete tweakable cipher using (e.g.) S-box and linear diffusion. Another approach, which we focus on, is building from ordinary block ciphers. There are several schemes [16] 24 that turn an $n$-bit block cipher into an $(n, n)$-bit tweakable cipher. However, they only have $O\left(2^{n / 2}\right)$-security 8 . Building a tweakable block cipher with better security has been considered as rather difficult (Liskov et al. mentioned it as an open problem [16]).

Our solution is simple and intuitive: changing keys depending on tweaks. This idea was possibly in mind of [16. However, to our knowledge it has not been seriously investigated 9 . Although our scheme is simple, its security proof needs some cares. Throughout this section, we occasionally write $E_{K}$ instead of $E$, if we need to specify the key we use.

Definition 5. For $E_{K}: \Sigma^{n} \rightarrow \Sigma^{n}$ with key $K \in \mathcal{K}$ and $F_{M K}: \Sigma^{m} \rightarrow \mathcal{K}$ with key $M K \in \mathcal{K}^{\prime}$, Tweak-dependent Rekeying (TDR) is an $(n, m)$-bit tweakable cipher, where its encryption is $\operatorname{TDR}[E, F](x, t)=E_{F_{M K}(t)}(x)$, and decryption is $\operatorname{TDR}[E, F]^{-1}(y, t)=E_{F_{M K}(t)}^{-1}(y)$. Here, the key of TDR $[E, F]$ is $F$ 's key, MK.

\footnotetext{
${ }_{8}$ In [10], tweakable ciphers having "security against exponential attacks" are proposed. They correspond to $(2 n, m)$-bit tweakable ciphers with $O\left(2^{n}\right)$-security, thus their security is up to the birthday bound of the block size.

${ }^{9}$ Liskov et al., said that a change of a tweak should be faster than a change of a key. This requirement is certainly desirable, however not mandatory one.
} 
Theorem 3. $\operatorname{Adv}_{\mathrm{TDR}[E, F]}^{\widetilde{\mathrm{sprp}}}(q, \tau) \leq \operatorname{Adv}_{F}^{\mathrm{prf}}(\eta, \tau+O(q))+\eta \operatorname{Adv}_{E}^{\mathrm{sprp}}(q, \tau+O(q))$, where $\eta \stackrel{\text { def }}{=} \min \left\{q, 2^{m}\right\}$.

Proof. Let R : $\Sigma^{m} \rightarrow \mathcal{K}$ be the URF. We have

$$
\operatorname{Adv}_{\operatorname{TDR}[E, F]}^{\widetilde{\operatorname{sprp}}}(q, \tau) \leq \operatorname{Adv}_{\mathrm{TDR}[E, F], \operatorname{TDR}[E, \mathrm{R}]}^{\widetilde{\mathrm{cca}}}(q, \tau)+\operatorname{Adv}_{\mathrm{TDR}[E, \mathrm{R}]}^{\widetilde{\operatorname{sprp}}}(q, \tau) .
$$

The first term of R.H.S. for Eq. (25) is clearly at most $\operatorname{Adv}_{F}^{\mathrm{prf}}(\eta, \tau+O(q))$, as we can evaluate $F$ or $\mathrm{R}$ on at most $\eta$ points. For the second term, the adversary can produce at most $\eta$ instances of $E$, and their keys are independent and uniform (as keys are generated from URF). For each sampled key, the adversary can query at most $q$ time 10 . Thus, the second term is at most $\eta \operatorname{Adv}_{E}^{\text {sprp }}(q, \tau+O(q))$ from the triangle inequality.

At first glance, TDR seems to provide a desirable security, since it simulates the tweakable URP in an intuitive way. However, this is not always the case. For example, when $\mathcal{K}=\Sigma^{n}$ and $m=n$, a simple attack using about $2^{n / 2}$ queries can easily distinguish TDR from $\widetilde{\mathrm{P}}_{n, n}$ : we first query a fixed plaintext with many distinct tweaks, and if a ciphertext collision is found for tweak $t$ and $t^{\prime}$, then query a new plaintext with tweaks $t$ and $t^{\prime}$ and see if the new ciphertexts collide again 11 .

Nevertheless, this scheme can have beyond-birthday-bound security if tweak length is not longer than the half of block length:

Corollary 4. Let $E_{K}$ be an $n$-bit block cipher with key $K \in \Sigma^{n}$. For $m<n / 2$, let $E^{\prime \prime}: \Sigma^{m} \rightarrow \Sigma^{n}$ be defined as $E^{\prime \prime}(x)=E\left(x \| 0^{n-m}\right)$. Then $\operatorname{Adv}_{\operatorname{TDR}\left[E, E^{\prime \prime}\right]}^{\widetilde{\operatorname{spr}}}(q, \tau)$ is at most $(\eta+1) \operatorname{Adv}_{E}^{\text {sprp }}(q, \tau+O(q))+\eta^{2} / 2^{n+1}$, where $\eta=\min \left\{q, 2^{m}\right\}$.

Here, $\operatorname{TDR}\left[E, E^{\prime \prime}\right]$ is secure if $2^{-(n-2 m)}$ is sufficiently small and $E$ is computationally secure, where "secure" means cca-advantage being much smaller than $2^{-m}$. Unfortunately, Corollary 4 does not tell us how large $q$ is admissible by itself, since the first term of the bound would not be negligible if $q$ is large. Nonetheless, as the first term is at least $\eta \tau+O(q) / 2^{n} \approx q / 2^{n-m}$ when $q \geq 2^{m}$ (achieved by the exhaustive key search, see [3]), we expect that $\operatorname{TDR}\left[E, E^{\prime \prime}\right]$ is computationally secure against attacks with $q \ll 2^{n-m}$ queries, if $E$ is sufficiently secure.

Practically, the big problem of TDR is the frequent key scheduling of $E$, as it may be much slower than encryption. Still, the negative impact on speed could be alleviated when on-the-fly key scheduling is possible.

Combining ENR and TDR. A combination of ENR and TDR provides a DBLC using an $n$-bit block cipher $E$. Let us consider combining the schemes

${ }_{10}$ A more elaborate analysis can significantly improve the second term of the bound. However, it requires some additional parameters to describe the adversary's strategy and thus the result would look rather complicated. We here make it simple.

11 This does not contradict with Theorem 3 the second term of the bound is at least $\eta(\tau+O(q)) /|\mathcal{K}|$, which is about $q^{2} / 2^{n}$ when $|\mathcal{K}|=2^{n}$, as pointed out by Bellare et al. 3]. 
from Corollaries 2 and 4 The resulting DBLC needs 4 calls of $E$ and two key schedulings of $E$. By assuming $\operatorname{Adv}_{E}^{\text {sprp }}(q, \tau) \approx q / 2^{n}$, the security bound of this DBLC is about $2 q / 2^{n-m}+8 q^{2} / 2^{(n+m) / 2}+2 q^{2} / 2^{2 n}+1 / 2^{n-2 m}$. Then the choice $m \approx n / 3$ achieves the security against $q \ll 2^{2 n / 3}$ queries for fixed $n$, which is the best possible for this combination. For example, if we use AES (i.e., $n=128$ ) and set $m=42$, the combined scheme's security is about 83.5-bit, assuming AES's security. Compared to the previous DBLCs having 64-bit security, the gain is not that large, but non-negligible. Of course, the security and efficiency of the resulting ENR would be greatly improved by using a better AES-based tweakable block cipher.

\section{Conclusion}

We described the extended Naor-Reingold (ENR), which converts an $n$-bit block tweakable block cipher into a $2 n$-bit block cipher. ENR has the beyond-birthdaybound security (for $n$ ) if underlying tweakable block cipher does, and has almost the same throughput as that of the tweakable block cipher. Hence, we have shown that a good (i.e., fast and secure) tweakable cipher implies a good double-blocklength cipher. We also described a way to convert an $n$-bit block cipher into tweakable one and achieves beyond-birthday-bound security based on the computational indistinguishability of the underlying block cipher. Unfortunately, this scheme has both theoretical and practical drawbacks due to its frequent rekeying. Thus, finding an efficient scheme without rekeying would be an important open problem.

Future Directions. It would be interesting to extend ENR to $m n$-bit block cipher for $m>2$ and make ENR tweakable, keeping beyond-birthday-bound security for $n$. Both problems can be basically solved by using ENR as a module of some known block cipher modes (e.g., CMC mode 12]) as they have $O\left(2^{n}\right)$-security with $2 n$-bit pseudorandom permutation. However, more efficient constructions may well be possible.

\section{Acknowledgments}

We would like to thank Thomas Ristenpart, Tetsu Iwata, and the anonymous referees for very helpful comments and suggestions. We also thank Debra L. Cook for suggesting references.

\section{References}

1. Aiello, W., Venkatesan, R.: Foiling Birthday Attacks in Length-Doubling Transformations - Benes: A Non-Reversible Alternative to Feistel. In: Maurer, U.M. (ed.) EUROCRYPT 1996. LNCS, vol. 1070, pp. 307-320. Springer, Heidelberg (1996)

2. Bellare, M., Desai, A., Jokipii, E., Rogaway, P.: A Concrete Security Treatment of Symmetric Encryption. In: Proceedings of the 38th Annual Symposium on Foundations of Computer Science, FOCS 1997, pp. 394-403 (1997) 
3. Bellare, M., Krovetz, T., Rogaway, P.: Luby-Rackoff Backwards: Increasing Security by Making Block Ciphers Non-invertible. In: Nyberg, K. (ed.) EUROCRYPT 1998. LNCS, vol. 1403, pp. 266-280. Springer, Heidelberg (1998)

4. Bellare, M., Rogaway, P.: The Security of Triple Encryption and a Framework for Code-Based Game-Playing Proofs. In: Vaudenay, S. (ed.) EUROCRYPT 2006. LNCS, vol. 4004, pp. 409-426. Springer, Heidelberg (2006)

5. Bernstein, D.J.: Stronger Security Bounds for Wegman-Carter-Shoup Authenticators. In: Cramer, R. (ed.) EUROCRYPT 2005. LNCS, vol. 3494, pp. 164-180. Springer, Heidelberg (2005)

6. Bertoni, G., Breveglieri, L., Fragneto, P., Macchetti, M., Marchesin, S.: Efficient Software Implementation of AES on 32-Bit Platforms. In: Kaliski Jr., B.S., Koç, Ç.K., Paar, C. (eds.) CHES 2002. LNCS, vol. 2523, pp. 159-171. Springer, Heidelberg (2003)

7. Carter, L., Wegman, M.: Universal Classes of Hash Functions. Journal of Computer and System Science 18, 143-154 (1979)

8. Schroeppel, R.: Hasty Pudding Cipher (1998), http://www.cs.arizona.edu/rcs/hpc

9. Crowley, P.: Mercy: A Fast Large Block Cipher for Disk Sector Encryption. In: Schneier, B. (ed.) FSE 2000. LNCS, vol. 1978, pp. 49-63. Springer, Heidelberg (2000)

10. Goldenberg, D., Hohenberger, S., Liskov, M., Schwartz, E.C., Seyalioglu, H.: On Tweaking Luby-Rackoff Blockciphers. In: Kurosawa, K. (ed.) ASIACRYPT 2007. LNCS, vol. 4833, pp. 342-356. Springer, Heidelberg (2007)

11. Goldreich, O.: Modern Cryptography, Probabilistic Proofs and Pseudorandomness. Algorithms and Combinatorics, vol. 17. Springer, Heidelberg (1998)

12. Halevi, S., Rogaway, P.: A Tweakable Enciphering Mode. In: Boneh, D. (ed.) CRYPTO 2003. LNCS, vol. 2729, pp. 482-499. Springer, Heidelberg (2003)

13. Iwata, T., Yagi, T., Kurosawa, K.: On the Pseudorandomness of KASUMI Type Permutations. In: Safavi-Naini, R., Seberry, J. (eds.) ACISP 2003. LNCS, vol. 2727. Springer, Heidelberg (2003)

14. Iwata, T., Kurosawa, K.: OMAC: One-Key CBC MAC. In: Johansson, T. (ed.) FSE 2003. LNCS, vol. 2887, pp. 129-153. Springer, Heidelberg (2003)

15. Iwata, T.: New Blockcipher Modes of Operation with Beyond the Birthday Bound Security. In: Robshaw, M.J.B. (ed.) FSE 2006. LNCS, vol. 4047, pp. 310-327. Springer, Heidelberg (2006)

16. Liskov, M., Rivest, R.L., Wagner, D.: Tweakable Block Ciphers. In: Yung, M. (ed.) CRYPTO 2002. LNCS, vol. 2442, pp. 31-46. Springer, Heidelberg (2002)

17. Luby, M., Rackoff, C.: How to Construct Pseudo-random Permutations from Pseudo-random functions. SIAM J. Computing 17(2), 373-386 (1988)

18. Patarin, J.: Security of Random Feistel Schemes with 5 or More Rounds. In: Franklin, M. (ed.) CRYPTO 2004. LNCS, vol. 3152, pp. 106-122. Springer, Heidelberg (2004)

19. Maurer, U.: Indistinguishability of Random Systems. In: Knudsen, L.R. (ed.) EUROCRYPT 2002. LNCS, vol. 2332, pp. 110-132. Springer, Heidelberg (2002)

20. Maurer, U., Pietrzak, K.: The Security of Many-Round Luby-Rackoff PseudoRandom Permutations. In: Biham, E. (ed.) EUROCRYPT 2003. LNCS, vol. 2656, pp. 544-561. Springer, Heidelberg (2003)

21. Mitsuda, A., Iwata, T.: Tweakable Pseudorandom Permutation from Generalized Feistel Structure. In: Baek, J., Bao, F., Chen, K., Lai, X. (eds.) ProvSec 2008. LNCS, vol. 5324, pp. 22-37. Springer, Heidelberg (2008) 
22. Naor, M., Reingold, O.: On the Construction of Pseudorandom Permutations: Luby-Rackoff Revisited. Journal of Cryptology 12(1), 29-66 (1999)

23. Patel, S., Ramzan, Z., Sundaram, G.: Towards Making Luby-Rackoff Ciphers Optimal and Practical. In: Knudsen, L.R. (ed.) FSE 1999. LNCS, vol. 1636, pp. 171-185. Springer, Heidelberg (1999)

24. Rogaway, P.: Efficient Instantiations of Tweakable Blockciphers and Refinements to Modes OCB and PMAC. In: Lee, P.J. (ed.) ASIACRYPT 2004. LNCS, vol. 3329, pp. 16-31. Springer, Heidelberg (2004),

http://www.cs.ucdavis.edu/ rogaway/papers/offsets.pdf

25. Krovetz, T.: Message Authentication on 64-Bit Architectures. In: Biham, E., Youssef, A.M. (eds.) SAC 2006. LNCS, vol. 4356, pp. 327-341. Springer, Heidelberg (2007)

26. Vaudenay, S.: On the Lai-Massey scheme. In: Lam, K.-Y., Okamoto, E., Xing, C. (eds.) ASIACRYPT 1999. LNCS, vol. 1716, pp. 8-19. Springer, Heidelberg (1999)

27. Wegman, M., Carter, L.: New Hash Functions and Their Use in Authentication and Set Equality. Journal of Computer and System Sciences 22, 265-279 (1981)

28. Yasuda, K.: A One-Pass Mode of Operation for Deterministic Message Authentication- Security beyond the Birthday Barrier. In: Nyberg, K. (ed.) FSE 2008. LNCS, vol. 5086, pp. 316-333. Springer, Heidelberg (2008)

\section{A Lemmas from Maurer's Methodology}

We describe some lemmas developed by Maurer [19] that we used in this paper. We assume that $F$ and $G$ are two random functions with the same input/output size; we define MESs $\mathcal{A}=a_{0} a_{1} \ldots$ and $\mathcal{B}=b_{0} b_{1} \ldots$ for $F$ and $G$. The $i$-th input and output are denoted by $X_{i}$ and $Y_{i}$ for $F$ (or $G$ ), respectively.

Lemma 1. (Lemma 1 (iv) of [19]) If $F|\mathcal{A} \equiv G| \mathcal{B}$ and $P_{a_{i} \mid X^{i} Y^{i-1} a_{i-1}}^{F} \leq P_{b_{i} \mid X^{i} Y^{i-1} b_{i-1}}^{G}$ holds for $i \geq 1$, then there exists an $M E S \mathcal{C}$ defined for $G$ such that $F^{\mathcal{A}} \equiv G^{\mathcal{B} \wedge \mathcal{C}}$.

Lemma 2. (Lemma 4 (ii) of [19]) Let $\mathbb{F}$ be the function of $F$ and $G$ (i.e., $\mathbb{F}[F]$ is a function that internally invokes $F$, possibly multiple times, to process its inputs). Here, $\mathbb{F}$ can be probabilistic, and if so, we assume $\mathbb{F}$ is independent of $F$ or $G$. If $F^{\mathcal{A}} \equiv G^{\mathcal{B}}$ holds, $\mathbb{F}[F]^{\mathcal{A}^{\prime}} \equiv \mathbb{F}[G]^{\mathcal{B}^{\prime}}$ also holds, where $a_{i}^{\prime}$ denotes an event that $\mathcal{A}$-event is satisfied for the time period $i$. For example, if $\mathbb{F}[F]$ always invoke $F$ c times for any input, then $a_{i}^{\prime}=a_{c i}$. $\mathcal{B}^{\prime}$ is defined in the same way.

Lemma 3. (Lemma 6 (ii) of [19]) If $F^{\mathcal{A}} \equiv G^{\mathcal{B}}$ holds for the attack class atk, then $\nu_{\text {atk }}\left(F, \overline{a_{q}}\right)=\nu_{\text {atk }}\left(G, \overline{b_{q}}\right)$ holds.

Lemma 4. (Lemma 6 (iii) of [19]) $\nu_{\text {atk }}\left(F, \overline{a_{q} \wedge b_{q}}\right) \leq \nu_{\text {atk }}\left(F, \overline{a_{q}}\right)+\nu_{\text {atk }}\left(F, \overline{b_{q}}\right)$.

Lemma 5. (An extension of Lemma 2 (ii) of [19]) If $F^{\mathcal{A}} \equiv G^{\mathcal{B}}$, then $F^{\mathcal{A} \wedge \mathcal{C}} \equiv$ $G^{\mathcal{B} \wedge \mathcal{C}}$ holds for any $M E S \mathcal{C}$ defined on the inputs and/or outputs. 


\section{B Proof of Equation (12)}

We focus on the indistinguishability between $\mathrm{DR}[0]$ and $\mathrm{R}_{2 n, 2 n}$. Let $\operatorname{dist}\left(X^{i}, Y^{j}\right)$ denote an event that there is no collision among $\left\{X_{1}, \ldots, X_{i}, Y_{1}, \ldots, Y_{j}\right\}$. Let $i$-th input (to DR[0] or $\left.\mathrm{R}_{2 n, 2 n}\right)$ be $X_{i} \stackrel{\text { def }}{=}\left(S E_{i}, T E_{i}\right) \in \Sigma^{n} \times \Sigma^{n}$, and $i$-th output be $Y_{i} \stackrel{\text { def }}{=}\left(U E_{i}, V E_{i}\right) \in \Sigma^{n} \times \Sigma^{n}$. We define events $i l_{q} \stackrel{\text { def }}{=} \operatorname{dist}\left(S E^{q}, \widehat{T E}^{q}\right)$ and $i r_{q} \stackrel{\text { def }}{=} \operatorname{dist}\left(\widehat{U E^{q}}, T E^{q}\right)$ and the corresponding MESs, $\mathcal{I} \mathcal{L}$ and $\mathcal{I R}$. For DR[0], let us analyze the conditional probability of $\widehat{Y_{q}}$ (which equals to $\widehat{U E}_{q}$ ), given $X^{q}=$ $x^{q}, Y^{q-1}=y^{q-1}$ and $i l_{q} \wedge i r_{q}$. Note that the inputs to $\mathrm{R}_{L}[0]$ are distinct from $i l_{q}$, which means $\widehat{Y^{q}}$ are independent and uniform. However, if $t e_{i}=t e_{j}$ for some $i \neq j, u e_{i} \neq u e_{j}$ must hold from $i r_{q}$. Thus, $\widehat{Y}_{q}$ is uniform over $\widehat{\mathcal{Y}}^{c}=\{0,1\}^{m} \backslash \widehat{\mathcal{Y}}$, where $\widehat{\mathcal{Y}} \stackrel{\text { def }}{=}\left\{u e_{i}: t e_{i}=t e_{q}, i=1, \ldots, q-1\right\}$. The remaining $(2 n-m)$ bits of $Y_{q}$ are uniform over $\Sigma^{2 n-m}$ from $i l_{q}$ and $i r_{q}$. For $\mathrm{R}_{2 n, 2 n}$, the set $\widehat{\mathcal{Y}}$ can be defined in the same way and $Y_{q}$ (given $X^{q}=x^{q}, Y^{q-1}=y^{q-1}$ and $i l_{q} \wedge i r_{q}$ ) is clearly uniformly distributed over $\widehat{\mathcal{Y}}^{c} \times \Sigma^{2 n-m}$. Thus we have

$$
P_{Y_{q} \mid X^{q} Y^{q-1} i l_{q} i r_{q}}^{\mathrm{DR}[0]}=P_{Y_{q} \mid X^{q} Y^{q-1} i l_{q} i r_{q}}^{\mathrm{R}_{2 n, 2 n}} .
$$

Next, we see that

$$
P_{i l_{q} i r_{q} \mid X^{q} Y^{q-1} i l_{q-1} i r_{q-1}}^{\mathrm{DR}[0]}\left(x^{q}, y^{q-1}\right)= \begin{cases}0 & \text { if } i l_{q} \text { is contradicted by } x^{q}, \\ \frac{\left|\hat{\mathcal{Y}}^{c}\right|}{2^{m}} & \text { otherwise. }\end{cases}
$$

holds true, as $i l_{q}$ is a function of $x^{q}$ and the conditional probability of $i r_{q}$ given $X^{q}=x^{q}, Y^{q-1}=y^{q-1}$, and $i l_{q} \wedge b_{q-1}$ is the probability of $\widehat{Y_{q}} \notin \widehat{\mathcal{Y}}$, where $\widehat{Y_{q}}$ is uniform given $i l_{q}$. Therefore, the conditional probability of $i r_{q}$ is $\left|\widehat{\mathcal{Y}}^{c}\right| / 2^{m}$ when $x^{q}$ satisfies $i l_{q}$. The same analysis also holds for $\mathrm{R}_{2 n, 2 n}$. Thus we have

$$
P_{i l_{q} i r_{q} \mid X^{q} Y^{q-1} i l_{q-1} i r_{q-1}}^{\mathrm{DR}[0]}=P_{i l_{q} i r_{q} \mid X^{q} Y^{q-1} i l_{q-1} i r_{q-1}}^{\mathrm{R}_{2 n, 2 n}}
$$

From Eqs. (26) and (28),

$$
\mathrm{DR}[0]^{\mathcal{I} \mathcal{L} \wedge \mathcal{I} \mathcal{R}} \equiv \mathrm{R}_{2 n, 2 n}^{\mathcal{I} \mathcal{L} \wedge \mathcal{I} \mathcal{R}} \text {, and } \mathrm{DR}[1]^{\mathcal{I} \mathcal{L}^{\prime} \wedge \mathcal{I} \mathcal{R}^{\prime}} \equiv \mathrm{R}_{2 n, 2 n}^{\mathcal{I} \mathcal{L}^{\prime} \wedge \mathcal{I} \mathcal{R}^{\prime}}
$$

is obtained. The latter equivalence is derived by symmetry, where $\mathcal{I} \mathcal{L}^{\prime}$ and $\mathcal{I} \mathcal{R}^{\prime}$ are defined by $i l_{q}^{\prime} \stackrel{\text { def }}{=} \operatorname{dist}\left(U D^{q}, \widehat{T D^{q}}\right)$ and $i r_{q}^{\prime} \stackrel{\text { def }}{=} \operatorname{dist}\left(\widehat{U D^{q}}, V D^{q}\right)$ (here, $i$-th input is $X_{i}=\left(U D_{i}, V D_{i}\right)$ and output is $\left.Y_{i}=\left(S D_{i}, T D_{i}\right)\right)$. From Eq. (29) and the independence of DR[0] and DR[1], the proof is completed.

\section{Proof of Equation (15)}

We abbreviate $\mathrm{R}_{n+m+1, n}$ and $\widetilde{\mathrm{P}}_{n, m}$ to $\mathrm{R}$ and $\mathrm{P}$, respectively. From the definition of GCF event $a_{q}$, it is easy to derive

$$
\langle\widetilde{\mathrm{P}}\rangle|\mathcal{A} \equiv \mathrm{R}| \mathcal{A} .
$$


Next, we have

$$
P_{a_{q} \mid X^{q} Y^{q-1} a_{q-1}}^{\langle\widetilde{\mathrm{P}}}\left(x^{q}, y^{q-1}\right)=1, \text { and } P_{a_{q} \mid X^{q} Y^{q-1} a_{q-1}}^{\mathrm{R}}\left(x^{q}, y^{q-1}\right)=1-\frac{\theta}{2^{n}},
$$

for any $x_{q}$ consistent with $a_{q}$ (given $x^{q-1}$ and $y^{q-1}$ ), since $\langle\widetilde{\mathrm{P}}\rangle^{\prime}$ 's output always keeps GCF, while R's output is uniform and thus has a chance to violate GCF. From Eq. (31), we get $P_{a_{q} \mid X^{q} Y^{q-1} a_{q-1}}^{\mathrm{R}} \leq P_{a_{q} \mid X^{q} Y^{q-1} a_{q-1}}^{\langle\widetilde{\mathrm{P}}\rangle}$. The proof is completed by combining this inequality, and Eq. (30), and Lemma 1 . 